\title{
Present Situation and Prospective of Camellia Nut Shells Utilization
}

\author{
Jinping Zhang*, Lisong Hu, Zhoulian Wang, Menghao Du, Xiaohua Yao \\ Research Institute of Subtropical Forestry, Chinese Academy of Forestry, Hangzhou, Fuyang, China \\ Email: *jinpingzhang@126.com
}

Received 6 September 2015; accepted 12 October 2015; published 15 October 2015

Copyright (C) 2015 by authors and Scientific Research Publishing Inc.

This work is licensed under the Creative Commons Attribution International License (CC BY). http://creativecommons.org/licenses/by/4.0/

c) (i) Open Access

\begin{abstract}
With the rapid development of Camellia oleifera industry, more and more Camellia nut shells have been produced, leading to serious environmental pollution problem. This paper reviews the works on the characterization of the physical and chemical properties of Camellia nut shells, active ingredient extraction, its application in chemical production, growing media and animal feed and its properties as a biomass. Based on the review, we proposed a novel all-component high value application strategy to covert camellia nut shells into high value biomasses, which could realize waste recycling and environmental protection.
\end{abstract}

\section{Keywords}

Camellia Nut Shell, Physical and Chemical Property Analysis, All-Component High Value Utilization, Utilization Status, Prospective Analysis

\section{Introduction}

Camellia oleifera is an important oleiferous tree species in southern China. It is one of the most valuable oleiferous species of Camellia and is considered, along with oil palm, olive and coconut, the four major oleifera tree species in the world (Zhuang, 2008). Camellia oleifera has been extensively cultivated in Yangtze River and the Pearl River basins of southern China, mostly in the provinces of Hunan, Jiangxi, Guangxi, Guangdong, Zhejiang, Fujian, Anhui, Guizhou, Yunnan, Henan, Hubei and so on. It can also be found in the provinces of Hainan, Sichuan, Shanxi, Jiangsu, Gansu and Taiwan (Hu, Tang, \& Liu, 2006). With the development of tea oil processing industry, the cultivation area of Camellia oleifera in China has reached $\sim 5.5$ million $\cdot \mathrm{hm}^{2}$ (Jiang, Chen, Zhuang, Pan, \& Zhang, 2011), which leads to an annual production of nearly 10 million tons of Camellia fruit 518,000 tons tea oil and nearly 8 million tons of camellia nut shells (Qiu, Chen, \& Hu, 2009; Shen, Kang, \& Chen, 2010;

"Corresponding author. 
Jiang, Ying, \& Chen, 2010). In recent years, the research on the extraction techniques and technology of tea oil extraction has made a significant progress. The processing and recycling of camellia nut shells have also been improved.

\section{Studies on the Utilization of Camellia Nut Shells}

\subsection{Physical and Chemical Property Analysis}

Camellia nut shell (Oiltea camellia or tea bags) is the shell of camellia oleifera fruit, which accounts for more than 60\% weight of the whole fruit (Qiu, Chen, \& Hu, 2009). Generally, Camellia nut shells are burned as fuels, causing a great waste of resources. Zhang et al. characterized the camellia nut shells of six most popular Camellia toleiferas including Camellia oleifera Abel, Camellia reticulate lindl, Camellia semiserrata Chi, Camellia mairei (Levl.) Melehior, Camellia meiocarpa Hu and Camellia polyodonta How (Zhang, Du, Yao, \& Hu, 2015). The results showed that cellulose, hemicellulose, lignin, ash and content of the organic extracts of the six Camellia nut shells were $13.87 \%$ - 20.95\%, 34.21\% - 49.34\%, 30.07\% - 36.23\%, 2.00\% - 4.75\% and 2.36\% $7.76 \%$, respectively. The contents of $\mathrm{K}, \mathrm{Na}, \mathrm{N}$ and organic carbon content were $7.6-13.80 \mathrm{mg} \cdot \mathrm{g}^{-1}, 0.02-0.26$

$\mathrm{mg} \cdot \mathrm{g}^{-1}, 2.91-4.42 \mathrm{mg} \cdot \mathrm{g}^{-1}, 44.4-48.6 \mathrm{mg} \cdot \mathrm{g}^{-1}$, respectively, and their cellulose crystallinity was $37.4 \%-62.3 \%$ (Zhang, Du, Yao, \& Hu, 2015). The thermal degradation of the six Camellia nut shells subjected to three stages including water loss in the temperature range of $30^{\circ} \mathrm{C}-150^{\circ} \mathrm{C}$ with a weight loss rate of $10 \%$, fast weight loss in the temperature range of $200^{\circ} \mathrm{C}-430^{\circ} \mathrm{C}$ with a weight loss rate of $60 \%$ and finally slow weight loss (Zhang, Du, Yao, \& Hu, 2015). Using Šatava-Šesták method, Ozawa integral method and Kissinger differential method, Gu et al. indicated that the pyrolysis process of camellia nut shell can be divided into three stages: drying, fast degradation and slow degradation, after analyzing Camellia nut shell from Hunan based on the thermogravimetric experiment data. Thirty kinds of reaction mechanism functions were adopted to analysis the kinetics of pyrolysis process of Camellia nut shell. The Avrami-Erofeev equation was the most reasonable model for camellia nut shell pyrolysis at different rates. The integral form was $[-\ln (1-a)]^{3}$ and the average activation energy was $79.59 \mathrm{~kJ} \cdot \mathrm{mol}^{-1}(\mathrm{Gu}$, Liu, Wang, Zhang, \& Zhou, 2015). Camellia nut shell extracts contain large amounts of saponins and polyphenols and low amount of flavonoids (Luo, Shen, Xiao, \& Chen, 2011). Xu et al. reported that the average tannin content in the Camellia nut shells from Guangxi province was $11.06 \%$ (Xu, $\mathrm{Chen}, \mathrm{Wang}$, Wu, \& Wu, 2009). Zhang et al identified 46 compounds in the Camellia nut shells $\mathrm{He}$ gas pyrolyzed at $590^{\circ} \mathrm{C}$ by $\mathrm{Py}-$ GC/MS method, including large amounts of rare bioactive medicine, spices and other ingredients (Zhang, Peng, Liu, Ma, Tan, Chen, \& Tian, 2008). Li et al. reported that the saponin content in the Camellia nut shells from Zhejiang province of China was 9.66\% (Li, Fang, Zhong, Fei, \& Luo, 2013). Li et al. analyzed six Camellia nut shells and regular Camellia from three different cultivation regions, including Camellia chekiangoleosa Hu, Camellia meiocarpa Hu, Camellia yuhsienensis Hu, Camellia semiserrata Chi, Camellia gigantocarpa Hu et Huang, Camellia oleifera Abel from Xianju, Sanjiang and Changshan, by spectrophotometry method. The results indicated that the extracts contain relatively high amounts of active compounds. The highest active ingredients were found in the Camellia oleifera Abel nut shells, with $>317 \mathrm{mg} / \mathrm{g}$ polysaccharide, $>35 \mathrm{mg} / \mathrm{g}$ flavono$\mathrm{ids},>292 \mathrm{mg} / \mathrm{g}$ saponin, $>252 \mathrm{mg} / \mathrm{g}$ polyphenol, $272.99 \pm 5.54 \mathrm{mg} / \mathrm{g}$ condensed tannin and $135.36 \pm 2.54 \mathrm{mg} / \mathrm{g}$ hydrolysis tannin content in its extract (Li, Fang, Zhong, Fei, \& Luo, 2013).

\subsection{Extraction and Application of Active Ingredients}

\subsubsection{Extraction and Application of Pigments}

The pigment in Camellia nut shells is an abundant, low cost and safe natural food coloring. However, the extraction and stability of the pigments in Camellia nut shells are rarely studied. Ling et al tested the toxicity of the brown pigment from Camellia nut shells and the results indicated that it had a chocolate brown color and was a safe food coloring (Ling, 1985). Qiu et al. extracted the brown pigment from Camellia nut shells by a microwave-assisted extraction method. The resulting brown pigment is a relatively stable natural pigment and can be widely used in the industries of food, medical and cosmetics and so on.

\subsubsection{Extraction and Application of Phenols}

\section{1) Extraction and application of tannin}

Tannin is a class of water-soluble compounds containing polyphenol hydroxyl groups. It can be classified hy- 
drolyzed tannins and condensed tannins and can be found in most plants. Camellia nut shells are an abundant natural tannin source due to its high tannin content. Solvents with a good solubility for tannin and ability to break the hydrogen bonds are required for the tannin extraction. Compared with water and alcohols, acetone is a better tannin extraction solvent ( $\mathrm{Li}$, Shen, \& Wu, 2013). Xu et al. extracted tannin from Camellia nut shells with the conventional and ultrasonic-assisted water extractions (Xu, Chen, Wang, $\mathrm{Wu}, \& \mathrm{Wu}, 2009)$. The preliminary study showed that the average tannin content in Camellia nut shells was $11.06 \%$ and the main ingredients were partially gallated proanthocyanidins. Yang et al extracted tannin from Camellia nut shells by a response surface optimized ultrasonic assisted distilled water extraction method and obtained $4.25 \%$ tannin (Yang, Liu, Li, \& Hu, 2012). Shen et al. purified tannin extracted from Camellia nut shells with macroporous resins and the purified 3-O-methylellagic acid 4'-O- $\beta$-D-glucopyranoside (MEAG) and ellagic acid (EA) contents were improved to $11.0974 \mathrm{mg} / \mathrm{g}$ and $2.0338 \mathrm{mg} / \mathrm{g}$, respectively, with a $85 \%$ recovery (Shen, Li, \& Wu, 2013).

Tannin has excellent physiological activities such as anti-bacterial, anti-viral, anti-allergic, anti-oxidation and so on. It has been widely used in leather production, food, household chemicals and mining industries. For example, ellagic acid can be used to reduce serum resisting levels and degrade the adipocytes resisting protein. It can also be used as an ingredient in cosmetics for skin whiting and scar repairing. In all, tannin is a promising new additives for food, nutrient supplements and cosmetics.

\section{2) Extraction and application of tea polyphenols}

Tea polyphenols is one of the most important and most widely distributed secondary metabolites. Tea polyphenols usually form complexes with biological molecules through hydrogen bonding. Solvents including acetone, methanol, ethanol and water can be used for polyphenol extraction. Chen et al obtained $20.17 \%$ polyphenol from Camellia nut shells with $60 \%$ acetone-water (Chen, Tang, \& Li, 2008). The yields increased to $45 \%$ with elution with $30 \%$ ethanol. Lin et al obtained $71.13 \mathrm{mg} / \mathrm{g}$ polyphenols from Camellia nut shells by refluxing extraction with ethanol under optimized conditions (Lin, Zhu, \& Wang, 2014). It has been tested that, among the Camellia nut shells from Guangxi, Jiangxi, Hunan, Zhejiang and Anhui provinces, the extract of Camellia oleifera Abel nut shells contains highest total phenol content (gallic acid equivalents $=234.90 \mathrm{mg} / \mathrm{g}$ ) and has the strongest antioxidant capacity (DPPH Clearance IC50 $=899.25 \mathrm{ug} / \mathrm{mL}$ and antioxidant capacity and anti-ascorbic acid equivalent $=12.91 \mathrm{mg} / \mathrm{g}$ ) (Zhang, Wang, \& Wu, 2010). The main active ingredients in Camellia polyphenols are gallic acid, ellagic acid and 3-O-methyl ellagic acid -4'-O- $\beta$-D-glucopyranose (MEAG) and so on. The content of gallic acid is the lowest with an extraction rate of $3.67 \mathrm{mg} / \mathrm{g}$ by alcohol extraction and an extraction rate of $8.94 \mathrm{mg} / \mathrm{g}$ by acid extraction (Chen, 2011; Chen, Shen, \& Zhao, 2014). The antioxidant capacity of polyphenol is affected by the extraction solvents and the amounts of the solvents and follows the order of ethyl acetate phase, butanol phase, the crude extract and water phase. The extracts have better abilities to remove $\mathrm{H}_{2} \mathrm{O}_{2}, \mathrm{O}_{2}$ and $\cdot \mathrm{OH}$ than its anti-ascorbic acid ability (Chen, Kang, Chen, \& Shen, 2011). Polyphenols from Camellia nut shells have effects of antioxidant, inhibition of cancer cell proliferation, hypoglycemic, lipid-lowering diet, prevention of prostate disease and so on. Gallic acid has effects of anti-tumor, anti-oxidation, anti-inflammatory, antibacterial, antiviral, hypoglycemic and so on and can be widely applied to the industries of medicine and nutrient supplement and so on.

\section{3) Extraction and application of flavonoid}

Flavonoid is a class of natural polyphenol. It is a series compounds consisting of two benzene rings with a phenolic hydroxyl group linked with each other through three carbon atoms $\left(\mathrm{C}_{6}-\mathrm{C}_{3}-\mathrm{C}_{6}\right)$. Shen et al. soaked Camellia nut shells in 30 times weight of $60 \%$ ethanol and extracted with the assistance of ultrasonic at $40^{\circ} \mathrm{C}$ for 45 $\mathrm{min}$, and repeated the extraction one more time. The recovery of total flavonoid extraction reached $97.34 \%$ and extracted amount was $1.709 \%$ of the shell weight (Shen, Jiang, \& Wang, 2008). Experimental studies show that the crude flavonoid extract from Camellia nut shells has a certain inhibition to the oxidation of cooking oils (Peng, 2012). Flavonoids have a variety of physiological functions and pharmacological effects, such as anti-oxidation, anti-cancer, anti-cardiovascular disease, anti-inflammatory, anti-viral, immune regulation and so on. Its extract has been applied to the products of food, medicine, cosmetics and so on (Shen, Jiang, \& Wang, 2008).

\subsubsection{Extraction and Application of Other Active Ingredients}

Saponins from Camellia nut shells can be used as a food preservative. Li et al. reported that the decoction of Camellia nut shells mixed with various amounts of bleach powder could be used for the storage of oranges harvested under desired conditions with controlled moisture ( $\mathrm{Li} \& \mathrm{Wu}, 1991)$. In addition, the decoction of Camellia nut shells can also be mixed with other reagents for the preparation of preservatives, pesticides, etc. 
The Camellia nut shell extracts contain a large amount of insoluble dietary fiber (IDF). Xie et al extracted the IDF from Camellia nut shells by base method under the optimum conditions (1:14 solid-liquid ratio in 0.35 $\mathrm{mol} / \mathrm{L} \mathrm{NaOH}$ at $80^{\circ} \mathrm{C}$ for $3 \mathrm{~h}$ ) and obtained $40.4 \%$ IDF with a purity of $91.52 \%$ (Xie, Chen, Chi, \& Liang, 2013). The water-holding capacity and swelling rate of the IDF at room temperature are $2.04 \mathrm{~g} / \mathrm{g}$ and $1.2 \mathrm{~mL} / \mathrm{g}$, respectively, suitable for conventional food applications (Xie, Chen, Chi, \& Liang, 2013). Therefore, the IDF in Camellia nut shells can be applied to nutrient supplement for weight loss, digestion, laxation, blood sugar control, and so on.

\subsection{Preparation and Application of Chemical from Camellia Nut Shells}

The abundant cellulose, hemicellulose and lignin in Camellia nut shells have been be used for the preparation of ethanol, xylose and vanillin, respectively, under optimum conditions (Zhu, Zhu, Jiang, Xu, Ouyang, \& Yu, 2013). Zhu et al. pretreated Camellia nut shells in $12 \% \mathrm{NaOH}$, at $120^{\circ} \mathrm{C}$ for $150 \mathrm{~min}$ with a liquid-solid ratio of 10.0 and obtained liquid and solid phases. The solid residue was enzymatically hydrolyzed and feminized, resulting in $17.35 \mathrm{~g} / \mathrm{L}$ ethanol with an $80.90 \%$ conversion rate. The partial hydrolysis and oxidation of the liquid phase produced $1758.63 \mathrm{mg} / \mathrm{L}$ xylooligosaccharides and $322.07 \mathrm{mg} / \mathrm{L}$ vanillin (Zhu, Zhu, Jiang, Xu, Ouyang, \& Yu, 2013). The hemicellulose was hydrolyzed to xylose (ketopentose) that was further hydrogenated to xylitol. Guo et al. sequentially pretreated Camellia nut shells with hot water, $\mathrm{NaOH}$ solution and dilute sulfuric acid, followed by the hydrolysis in $1 \%-2 \%$ sulfuric acid for the preparation of xylose (Guo, 1992). The xylose solution was then neutralized with lime emulsion, decolorized on active carbon, concentrated, cleaned by ion exchange, concentrated, crystallized, centrifuged and dried to obtain a xylose crystalline with a purity over $97.5 \%$. Xu from Lishui city, Zhejiang province hydrolyzed the pentosan from Camellia nut shells to xylose and hydrogenated xylose to xylitol with acceptable quality. Camellia nut shells can be mixed with pig manure as a raw material to produce biogas by the fermentation under anaerobic condition in batches or semi-continuous batches. A maximum yield of $2025 \mathrm{~mL} / \mathrm{dL}$ was obtained with 1:1 manure/shell ratio (Yu, 2013). Xylose prepared from Camellia nut shells can be used in the food, chemical, and leather industries, such as the production of xylitol, animal feed yeast, furfural and sweeteners. Xylitol is a polyol with five hydroxyl groups. It is a white fine crystal and can be used as the raw materials for the production of plasticizers, paint, chemical fiber additives and so on. It has also been applied to the light industrial products, such as toothpaste, cigarettes, paper and so on. Xylitol is a best-selling medicine for the treatment of diabetes. Camellia nut shells can be processed by various methods, such as fermentation, hydrolysis, enzymatic hydrolysis and so on, to prepare biomasses. For example, the ethanol prepared from Camellia nut shells is an important organic solvent and raw material for the synthesis of other solvents. It has also been used as fuel, disinfectants, preservatives, beverages, food additives, and so on. Vanillin is an important spice and can be used as a fixative agent and flavoring agents. It has also been applied to pharmaceuticals.

Furfural (furaldehyde) is a heterocyclic organic compound. It is a colorless or transparent amber oily liquid and generally produced by the hydrolysis of pentosan followed by dehydration and cyclization (Liu, Li, Wang, \& Hu, 2013). One-step and two-step methods have been used for the production of furfural. One-step method includes sulfuric acid method, modified sulfuric acid method, hydrochloric acid method, acetic acid method and inorganic method. Among all crops, Camellia nut shells contain the highest amount of furfural $(\sim 19 \%)$. Therefore and can be used as the raw material for the production of furfural. Ye et al. obtained $0.137 \mathrm{~mol} / \mathrm{L}$ furfural from Camellia nut shells by sulfuric acid catalyzed hydrolysis with $230 \mathrm{~mL} 2 \mathrm{~mol} / \mathrm{L}$ sulfuric acid at $155^{\circ} \mathrm{C}(Y e$, $\mathrm{Hu}, \& \mathrm{He}, 2015$ ). Yang et al. extracted furfural with a modified sulfuric acid method (sulfuric acid with bisulfite, phosphate, calcium phosphate, etc.) with a solid-liquid ratio of $5.96 \mathrm{~mL} / \mathrm{g}$ at $140^{\circ} \mathrm{C}$ for $3.33 \mathrm{~h}$ and obtained $1.93 \%$ furfural (Yang, Liu, Li, Xu, \& Hu, 2011). Furfural in foods can be directly used as a preservative. It can be oxidized and reduced to synthesize advanced preservatives added in chewing gum, candy, cereal to prevent dental caries. It can also be used in the production of pesticides, such as the production of maleic acid or maleic anhydride by oxidation and Marathon by esterification for killing rice leafhopper, aphid, scale insects and mites. Furfural with a variety of reagents can be used to synthesize multiple pharmaceutical products, such as antibacterial agents, anticancer drugs, anesthetic and anti-inflammatory agents and so on. Its double bond, ether bond and aldehyde group can be oxidized to generate various chemical products, such as the furan resin with good corrosion resistance and thermal stability. In all, furan ring and the aldehyde group of furfural provide extensive potential for its application in the preparation of various chemicals and their derivatives (Jiang, 2009). Hy- 
drochloric acid is a common pickling reagent. Small amount of corrosion inhibitor in the pickling solution can slow corrosion process. Corrosion inhibitors of inorganic reagent or synthetic chemicals have a variety of disadvantages, such as toxicity, high cost and so on. Furfural has been used as a high efficient, low cost, multiple controlling and environment-friendly corrosion inhibitor (Liu, Li, Wang, \& Hu, 2013).

Potassium carbonate is an important raw material for the industries of chemical, pharmaceutical and light industry. It has been widely used in the industries of light bulbs, electroplating, pharmaceutical industries and so on. Feng et al. extracted potassium carbonate and potassium pyrophosphate crystalline with high purities from Camellia nut shells (Feng \& Xu, 2002). Potassium pyrophosphate can be used to substitute potassium cyanide for non-toxic electroplating. In Sanjiang county of Guangxi province, Camellia nut shells have been used to produce composite fertilizer-monopotassium phosphate with a process of Camellia nut shells ashing $\rightarrow$ dehydrating $\rightarrow$ boiling dry $\rightarrow$ high temperature calcination $\rightarrow$ water dissolving ( $7-10$ days) $\rightarrow$ neutralizing with phosphoric acid solution $\rightarrow$ water removing by centrifuge. The resulting water-soluble and high efficient composite fertilizer contains $30 \%$ potassium chloride and $50 \%$ phosphorus pentoxide.

\subsection{Utilization in Bio-Energy and Bio-Material}

Cellulose, hemicellulose and lignin rich in hydroxyl group are the major components of Camellia nut shells. Theoretically, these compounds can be used to substitute petroleum-based polyether polyols in the nucleophilic addition reactions with isocyanate to prepare polyurethane materials. Zhang et al. liquefied Camellia nut shells at $130^{\circ} \mathrm{C}-170^{\circ} \mathrm{C}$ for 120 min with PEG400 and glycerol as the liquefying agent and $3 \%$ sulfuric acid as a catalyst. The liquefaction rate reached $98.12 \%$ and the resulting liquefied product contains ethers and large amount of esters that can substitute petroleum-based polyether or polyester for the preparation of polyurethane polyols (Zhang, Du, Hu, Fei, \& Fang, 2012).

The briquettes fuel has been made which has certain specifications and dimensions, by crushing and compressing Camellia nut shell using biomass compression molding equipment (Wei, 2013). Wei discussed the mechanism of Camellia nut shell molding, and designed a set of processing equipment with can with an theoretical annual output of 40,000 tons Camellia nut shell pellet fuel. The work can provide a theoretical basis for the technical improvement of biomass compression molding and the design optimization of the molding equipments. Zhu et al. has prepared machine-made carbon with the shells of camellia (Camellia oleifera), chestnut tea (Castanea mollissima) and walnut (Caryacathayensis) as raw materials, which will provide a basis for high efficient energetic utilization of epicarps resources and the development of deep processing products of carbon derivatives (Zhu, Zhuang, Pan, Chen, \& Zhang, 2013).

\subsection{Extraction and Application of Absorbent}

Camellia nut shells are carbon-rich and a good raw material for the preparation of activated carbon. It can be produced from Camellia nut shells by steam/flue gas activation method, phosphoric acid activation method, zinc chloride activation method, potassium hydroxide activation method and so on. Sun et al prepared the microporous and mesoporous activated carbon with excellent adsorption performance by steam activation and phosphoric acid re-activation method, which can be used for the purification of the water containing complex contaminations (Sun, Jiang, He, Lin, Cui, \& Lu, 2010). Jiang et al. prepared activated carbon powder from Camellia nut shells by microwave heating method with phosphoric acid as the activator. The activated carbon showed 226 $\mathrm{mg} / \mathrm{g}$ absorption of methylene blue and $100 \%$ caramel decolorization ratio (Jiang, Chen, Zhuang, Pan, \& Zhang, 2011). Zhou et al. prepared activated carbon under reduced pressure by zinc chloride method, which showed an $1120 \mathrm{mg} / \mathrm{g}$ absorption rate for iodine and $373.16 \mathrm{mg} / \mathrm{g}$ absorption rate for methylene blue (Zhou, Qiu, Shen, \& Huang, 2015). Song et al. studied the absorption properties of the ground Camellia nut shells for the crystal violet in wastewater and found that it showed 95\% removal of crystal violet after recycled for 7 times (Song, Guo, $\&$ Yan, 2014).

The activated carbon prepared from Camellia nut shells is a good adsorbent material with a large surface area, good mechanic strength, homogenous size, high porosity, high absorption, and other good properties. It can be used for the effective absorption of free chlorine, phenol, sulfur, oil, gum, pesticide residues and other organic pollutants, organic solvent recycling and so on. The activated carbon can also be applied to the industries of pharmaceutical, petrochemical, production of sugar and beverages, alcohol purification and environmental protection, as well as bleaching, refining and purification of organic solvents and sewage treatment. It can also be 
used for air purification and odor absorption, such as the deodorants for refrigerators, toilet and so on. In addition, the untreated Camellia nut shells can be used as a bio-absorbent for the dyes in wastewater.

\subsection{Application in Cultivation Substrate}

Camellia nut shells contain a variety of nutrients and chemical components and is a promising cultivation substrate with very good market prospects. For example, sawdust with 30\% - 40\% Camellia nut shells were used for the shiitake cultivation and no significance was observed on the production and quality (Lv, Ying, Li, Xue, Chen, \& Fan, 2014; Ying, Lv, Xue, \& Li, 2015). The cultivation of Hericium erinaceus in the sawdust with $20 \%$ and $40 \%$ Camellia nut shells can improve its growth. Its production was significantly increased $29.2 \%$ in the cultivation substrate containing 63\% Camellia nut shells (Lv, Li, Ying, Xue, Ye, Wang, \& Zhu, 2011). Partially Camellia nut shell powder substituted cotton seed hull cultivation substrate can be used for the cultivation of Enokitake. However, the growth rate, production and biotransformation rate of Enokitake is decreased with the increase of substitution rate when the substitute rate is over $40 \%$ (Chen \& Mao, 2015). Chen et al mixed the dried Camellia nut shell powder and sawdust at different ratios for the cultivation of black fungus and found that the production of black fungus was significantly improved and the highest production was obtained with 2:8 ratio of Camellia nut shell powder to sawdust (Chen, 2013). Luo et al. prepared a soilless cultivation substrate by the fermentation of Camellia nut shells with nitrogen and microbes (Luo, Tan, \& Peng, 2011). Zhang et al developed a cultivation substrate for Dendrobium nobile with a composition of 30 - 70 Camellia nut shells, 10 - 40 Camellia meal, 10 - 30 organic filler and 10 - 20 biomass polyurethane release fertilizer (Zhang, Wang, Du, Yao, $\& \mathrm{Hu}, 2015)$. The cultivation substrate was able to retain and absorb water and provide active ingredient for the growth of Dendrobium nobile. The polysaccharide content of the 1 - 2-year-old Dendrobium nobile grew in the substrate reached as high as $40 \%-50 \%$. Qu et al. investigated the effects of Camellia nut shell substrate on the growth of spring Dendrobium and the results indicated that pure Camellia nut shell can potentially replace sphagnum as the cultivation substrate for Dendrobium nobile in central China (Qu, Qiu, Wu, Liu, \& Wang, 2012). In addition, Camellia nut shells can also be used as the cultivation substrates for other edible fungi, medicinal plants and so on.

\section{Application Study Tendency of Camellia Nut Shells}

At present, the study of the utilization of Camellia nut shells has made some significant progresses. The extraction techniques for pigments, tannin and polyphenols and the preparation of activated carbon and chemicals from Camellia nut shells have been well developed. However, the most utilization of Camellia nut shells is limited in the laboratory and lacks of variety. Therefore, all-component high-value utilization has not been realized, which limits its application in industrial scale. Particularly, 1) the physical and chemical properties of Camellia nut shells have not been well studied. Only the physical and chemical properties of some Camellia nut shells have been reported. 2) Studies have been focused on utilizing Camellia nut shells as a raw material, active ingredient extraction, and chemical conversion. It is based on single product extraction and preparation of chemicals from some components in Camellia nut shells. The large amounts of residues produced from these ingredient extraction and chemical production have not been studied. 3) The function mechanism of the active ingredients from Camellia nut shells has been focused on its extract as lipid-lowering diet, antioxidant and anti-cancer and so on. The applications of saponins, flavonoids, volatile oils, polyphenols, polysaccharides and other ingredients of Camellia nut shells have been rarely studied. These active ingredients can be potentially used for the development of new medicines and nutrient supplements. 4) The application of Camellia nut shells in bio-feed and cultivation substrates have been only focused on their effects on the growth rates of animals, plants and edible fungi mycelial. The effects of the active ingredients such as tannins, pigments, proteins, polysaccharides, phenols and so on, on the growth and metabolism of animals, plants and edible fungus have been rarely investigated.

Based on the review of the current studies on Camellia nut shells, we proposed the study strategies for the utilization of the Camellia nut shells in the future.

First, due to the lack of the knowledge of the physical and chemical properties of Camellia nut shells, further work should be focused on determining the physical and chemical properties of all Camellia nut shells to establish a physical and chemical property database of Camellia nut shells. The database will provide a foundation for the characterization and utilization of Camellia nut shells. 
Second, future work should be focused on the systematic and comprehensive study all-component high-value utilization. Based on the physical and chemical properties of various kinds of Camellia nut shells, the extraction sequence of active ingredient shall be established. The extracts and residues shall be subjected continuous tracking and analysis to determine their physical and chemical properties that can be used for further exploration of their applications. For example, the extraction solvents can affect the physical and chemical properties, extraction process kinetics and applications of the extracts or the chemical products. High-temperature and highpressure method or solvent can be used for complete liquefaction of the residue. The liquefied substance can be used to produce chemicals. In all, an all-component high-value conversion and application system would ultimately assist to realize the all-component utilization of Camellia nut shells.

Third, from the perspective of nutritional, metabolic and toxicological analysis, the future study should be focused on the effect of the pretreatment and chemical ingredients of the Camellia nut shells as cultivation substrates and bio-feed on the metabolisms and nutrient contents of animals, plants, edible mushrooms and so on.

\section{Conclusion}

All in all, further exploration of the all-component high value utilization of Camellia nut shells is highly desired. Based on the physical and chemical properties of Camellia nut shell, a regulation mechanism of sequential active ingredient extraction, separation, structural characterization, and extraction techniques can provide a fundamental evidence for its high efficiency active ingredient extraction. All components in the extraction residues can be further converted to chemicals, which complete the theory of the utilization system of Camellia nut shells. The conversion mechanism and process micro-controlled production mechanism can be established. Based on the effects of the chemical components of Camellia nut shells on the growth, nutrient and metabolism of edible fungus, animals and plants, the pre-treatment methods and preparation technology system can be established to provide experimental evidence for the production of cultivation substrates and bio-feed. These proposed research strategies will completely solve the bottlenecks in the all-component high-value utilization of Camellia nut shells in industrial scale.

\section{Acknowledgements}

The authors are grateful for the financial support from Research Institute of Sub-tropical Forestry, Chinese Academy of Forestry, Fund No. RISF6130101076 and Provincial Department of Science and Technology of Zhejiang, China, Grant No. 2013C32096.

\section{References}

Chen, Q. M. (2013). Experimental Cultivation of Jew Ear Fungi on Camellia oleifera Shell Substrate. Non-timber Forest Product, 11, 61.

Chen, Q. P. (2011). Effects of Functional Factors in the Hull of Camellia oleifera Abel: On Obesity and Dyslipidemia. Ph.D. Thesis, Hangzhou: Zhejiang University.

Chen, Q. P., Shen, J. F., \& Zhao, Y. C. (2014). Optimzing Acid Extraction Technology of Gallic Acid from Fruit Hull of Camellia oleifera Abel. Food Science and Technology, 39, 195-198.

Chen, X. P., \& Mao, X. W. (2015). Experimental Cultivation of Enokitake on Camellia oleifera Shell Partially Substituted Cottonseed Hulls Substrate. Edible and Medicinal Mushrooms, 23, 201-202.

Chen, Y. L., Tang, L., \& Li, H. B. (2008). Study on the Technology of Enriching Polyphenol from Camellia oleifera Abel Peel by Macroporous Resin. Shi Zhen Guo Yi Guo Yao, 19, 1369-1370.

Chen, Y. Q., Kang, H. Q., Chen, Q. P., \& Shen, J. F. (2011). Antioxidant Activity of Extracts from the Fruit Shell of Camellia oleifera. Science Silvae Sinicae, 47, 20-24.

Feng, Y. L., \& Xu, R. Z. (2002). Producing Potassium Carbonate and Potassium Pyrophosphate with Endoconches of Hickory. Bancoul Nut and Oiltea: Journal of Zhejiang Shuren University, 2, 71-73.

Gu, J., Liu, B., Wang, L., Zhang, Q. S., \& Zhou, J. B. (2015). Research on Pyrolysis Process and Kinetics of Camellia Shell Using Šatava-Šesták Method. Science Technology and Engineering, 15, 36-41.

Guo, X. Y. (1992). Production of XYLOSE from Camellia oleifera Shells. Yunnan Chemical Technology, 3, 55-57.

Hu, F. M., Tang, X. F., \& Liu, H. M. (2006). Main Economic Tree Cultivation and Utilization in China. Beijing: Chinese Forestry Publishing House. 
Jiang, J. F. (2009). Production and Application of Furfural. Chemical Engineering \& Equipment, 10, 137-139.

Jiang, T. J., Ying, T. J., \& Chen, Q. P. (2010). Total Flavonoids and Antioxidant Activity of Different Solvent Extracts from Shells of Oiltea (Camellia oleifera) Seed. Journal of Chinese Institute of Food Science and Technology, 10, 93-99.

Jiang, Y. T., Chen, S. W., Zhuang, X. W., Pan, X., \& Zhang, J. L. (2011). Preparation of Activated Carbon from Oil Camellia Nutshell by Phosphoric Acid Activation Using Microwave Heating. Biomass Chemical Engineering, 45, 34-36.

Li, D. S., \& Wu, K. H. (1991). The Preservative Effect of the Decoction of Camellia oleifera Shell on the Citrus reticulata Blanco (First Report). Zhejiang Citrus Reticulata Blanco, 1, 37-38.

Li, H., Fang, X. Z., Zhong, H. Y., Fei, X. Q., \& Luo, F. (2013). Tea Saponin Contents Distribution in Maturation Process of Camellia Fruit. Cereals \& Oils, 26, 24-26.

Li, L. M., Shen, J. F., \& Wu, X. Q. (2013). Comparative Study on Contents of Bioactive Substance and Antioxidant Activities of Eight Kinds of Camellia Fruit Hull Extracts. Journal of the Chinese Cereals and Oils Association, 28, 41-47.

Lin, G. S., Zhu, J. C., \& Wang, X. D. (2014). Study on Extracting Total Polyphenol of Camellia Fruit Shells by Reflux Technology. Applied Chemical Industry, 43, 971-974.

Ling, C. (1985). The Toxicity Test of the Domestic Natural Brown Pigment of Camellia oleifera Shells. Chinese Journal of Preventive Medicine, 19, 16.

Liu, J. P., Li, Q. Q., Wang, S. Y., \& Hu, L. (2013). Furfural Extracted from Hickory Hull Optimized by Response Surface Methodology and Its Inhibition Performance. Corrosion \& Protection, 34, 790-795.

Luo, J., Tan, X. F., \& Peng, Y. (2011). A Study on the Composting Technology for Camellia Shell Medium. Acta Agriculturae Universitatis Jiangxiensis, 33, 712-718.

Luo, X. W., Shen, J. F., Xiao, R. X., \& Chen, Z. H. (2011). Determination of Secondary Metabolism in Extracts from Fruit Shell of Camellia oleifera Abel. Science and Technology of Food Industry, 32, 451-453.

Lv, M. L., Li, L. L., Ying, G. H., Xue, Z. W., Ye, R. H., Wang, W. P., \& Zhu, Q. (2011). Cultivation of Hericium erinaceus Using Powdered Camellia oleifera (Oil-Seed Camellia) Seed Coats and Chestnut Shells. Acta Edulis Fungi, 18, 6-8.

Lv, M. L., Ying, G. H., Li, L. L., Xue, Z. W., Chen, X., \& Fan, L. M. (2014). Experimental Study on High Temperature Type Lentinus edodes Cultivation with Camellia oleifera Shell. Edible Fungi of China, 33, 33-34.

Peng, L. (2012). Camellia Shell Flavonoids on Antioxidant Activities of Edible Oils. Food Research and Development, 33, 210-204.

Qiu, H. Y., Chen, J. M., \& Hu, J. R. (2009). Microwave Extraction and Stability of Brown Pigment from Camellia oleifera Shells. Food Science, 30, 198-202.

Qu, Y., Qiu, S., Wu, J., Liu, Z. D., \& Wang, C. Y. (2012). Effect of Camellia Shell on the Growth and Development of Nobile-Type Dendrobium. Advances in Ornamental Horticulture of China, 377-383.

Shen, J. F., Jiang, T. J., \& Wang, X. Q. (2008). Optimizing Extraction Technology of Total Flavones from Shell of Camellia oleifera Abel. Journal of the Chinese Cereals and Oils Association, 23, 104-106.

Shen, J. F., Kang, H. Q., \& Chen, Y. Q. (2010). The Study of the Extraction of Polysaccharides from Camellia oleifera Shells and Their Anti-Oxidation Effect. Journal of The Chinese Cereals and Oil Associations, 25, 51-54.

Shen, J. F., Li, L. M., \& Wu, X. Q. (2013). Purification of Tannins from Shell of Camellia oleifera Abel by Macroporous Resins. Journal of Chinese Institute of Food Science and Technology, 13, 115-121.

Song, D. Y., Guo, H. Q., \& Yan, L. S. (2014). Adsorptive Property of Crystal Violet on Camellia oleifera Shell in Aqueous Solution. Chinese Journal of Environmental Engineer, 8, 5129-5134.

Sun, K., Jiang, J. C., He, Y., Lin, Y. S., Cui, D. D., \& Lu, X. C. (2010). Preparation of Activated Carbon from Camellia oleifera Shell and Its Mesoporous Structure Modification. Chemistry and Industry of Forest Products, 30, $24-28$.

Wei, W. (2013). Researches on Molding Mechanisms and Complete Set of Equipment of Fruit Shell of Camellia oleifera Abel Fuel. Master's Thesis, Nanchang: Nanchang Hangkong University.

Xie, Y., Chen, S. Q., Chi, X. L., \& Liang, Y. C. (2013). Extraction of Insoluble Dietary Fiber from Hull of Camellia oleifera Abel and Its Physicochemical Properties. Journal of Zhejiang University, 39, 149-154.

Xu, M., Chen, J. H., Wang, Y. M., Wu, D. M., \& Wu, Z. S. (2009). Preliminary Report of Extraction and Analysis of Tannins from Camellia oleifera Fruit Shell. Chemistry and Industry of Forest Products, 29, 187-191.

Yang, X. M., Liu, J. P., Li, Q. Q., \& Hu, L. (2012). Optimization of Ultrasonic Assisted Tannins Extraction Technology from Camellia oleifera Abel Nutshell by Response Surface Methodology. Science and Technology of Food Industry, 33, 215-218.

Yang, X. M., Liu, J. P., Li, Q. Q., Xu, P. F., \& Hu, L. (2011). Optimization of Furfural Extraction from Camellia oleifera Abel Nutshell by Response Surface Methodology. Transactions of the Chinese Society for Agricultural Machinery, 42, 151-155. 
Ye, Z., Hu, C. R., \& He, D. P. (2015). Optimization of the Furfural Preparation Technology from Camellia Sees Shell by Response Surface Methodology. Food Science and Technology, 40, 218-223.

Ying, G. H., Lv, M. L., Xue, Z. W., \& Li. L. L. (2015). Experimental Study on Lentinus edodes Cultivated with Camellia oleifera Shell in Autumn. Edible Fungi of China, 34, 19-21.

Yu, Z, Z. (2013). Study on Co-Digestion of Camellia Shell and Pig Manure for Anaerobic Digestion. Master's Thesis, Nanchang: Nanchang University.

Zhang, D. Q., Peng, W. X., Liu, Q. M., Ma, Q. Z., Tan, X. F., Chen, H. P., \& Tian, H. (2008). Py-GC/MS Analysis to Estimate High-Grade Resource Recovery of Camellia oleifera Shell. Journal of the Chinese Cereals and Oils Association, 23, 161-165.

Zhang, J. P., Du, M. H., Hu, L. S., Fei, X. Q., \& Fang, X. Z. (2012). Thermochemical Liquefaction Based on Camellia Nut Shell and Production of Vegetal Polyalcohol. World Automation Congress, 24-28, 1949-1952.

Zhang, J. P., Du, M. H., Yao, X. H., \& Hu, L. S. (2015). A Cultivation Substrate for Dendrobium nobile and Its Preparation. China Patent No. 2015103964871.

Zhang, J. P., Wang, Z. L., Du, M. H., Yao, X. H., \& Hu, L, S. (2015). Physicochemical Properties of Camellia Nut Shell and Its Thermal Degradation Characteristics. Bioresources, 10, 647-659.

Zhang, L. L., Wang, Y. M., \& Wu, D. M. (2010). Comparisons of Antioxidant Activity and Total Phenolics of Camellia oleifera Abel Fruit Hull from Different Regions of China. Journal of Medicinal Plants Research, 4, 1407-1413.

Zhou, Q., Qiu, H. H., Shen, J., \& Huang, M. (2015). Preparation of Activated Carbons from Oiltea Shell Promoted by Zinc Chloride in Vacuum. Fine Chemicals, 32, 500-504.

Zhu, H. R., Zhuang, X. W., Pan, X., Chen, S. W., \& Zhang, J. L. (2013). Combustion Performance of Charcoal Briquettes Made from Three Kinds of Epicarps. Journal of Zhejiang A \& F University, 30, 90-94.

Zhu, J. J., Zhu, Y. Y., Jiang, F. X., Xu, Y., Ouyang, J., \& Yu, S. Y. (2013). An Integrated Process to Produce Ethanol, Vanillin, and Xylooligosaccharides from Camellia oleifera Shell. Carbohydrate Research, 382, 52-57. http://dx.doi.org/10.1016/j.carres.2013.10.007

Zhuang, R. L. (2008). China Camellia (2nd ed.). Beijing: China Forestry Publishing House. 